\title{
Loss of DBC1 (CCAR2) affects TNF $\alpha$-induced lipolysis and Glut4 gene expression in murine adipocytes
}

\author{
Ashley A Able1,2, Allison J Richard1 and Jacqueline M Stephens ${ }^{1,2}$ \\ 'Adipocyte Biology Laboratory, Pennington Biomedical Research Center, Baton Rouge, Louisiana, USA \\ 2Department of Biological Sciences, Louisiana State University, Baton Rouge, Louisiana, USA \\ Correspondence should be addressed to J M Stephens: jsteph1@lsu.edu
}

\begin{abstract}
STAT5A (signal transducer and activator of transcription $5 \mathrm{~A}$ ) is a transcription factor that plays a role in adipocyte development and function. In this study, we report DBC1 (deleted in breast cancer 1 - also known as CCAR2) as a novel STAT5A-interacting protein. $\mathrm{DBC} 1$ has been primarily studied in tumor cells, but there is evidence that loss of this protein may promote metabolic health in mice. Currently, the functions of DBC1 in mature adipocytes are largely unknown. Using immunoprecipitation and immunoblotting techniques, we confirmed that there is an association between endogenous STAT5A and $\mathrm{DBC} 1$ proteins under physiological conditions in the adipocyte nucleus that is not dependent upon STAT5A tyrosine phosphorylation. We used siRNA to knockdown DBC1 in 3T3-L1 adipocytes to determine the impact on STAT5A activity, adipocyte gene expression and TNF $\alpha$ (tumor necrosis factor $\alpha$ )-regulated lipolysis. The loss of DBC1 did not affect the expression of several STAT5A target genes including Socs3, Cish, Bcl6, Socs2 and Igf1. However, we did observe decreased levels of TNF $\alpha$-induced glycerol and free fatty acids released from adipocytes with reduced DBC1 expression. In addition, DBC1-knockdown adipocytes had increased Glut4 expression. In summary, DBC1 can associate with STAT5A in adipocyte nucleus, but it does not appear to impact regulation of STAT5A target genes. Loss of adipocyte DBC1 modestly increases Glut4 gene expression and reduces TNF $\alpha$-induced lipolysis. These observations are consistent with in vivo observations that show loss of DBC1 promotes metabolic health in mice.
\end{abstract} Key Words

- DBC1

- adipocytes

- gene expression

- lipolysis

Journal of Molecular Endocrinology (2018) 61, 195-205

\section{Introduction}

Signal transducer and activator of transcription $5 \mathrm{~A}$ (STAT5A) is a transcription factor that utilizes the janus kinase/signal transducer and activator of transcription (JAK/STAT) pathway to mediate the biological actions of a variety of hormones and cytokines. In vivo and in vitro approaches have demonstrated that STAT5A has a prominent role in adipogenesis (Teglund et al. 1998, Floyd \& Stephens 2003, Stewart et al. 2011, Wakao et al. 2011, Tse et al. 2013). Growth hormone (GH) is a primary activator of STAT5A in adipocytes. Upon GH activation, STAT5A can activate or repress several genes including suppressor of cytokine signaling 3 (Socs3), cytokine-inducible SH2-containing protein (Cish), B-cell lymphoma 6 (Bcl6), fatty acid synthase (Fasn), adiponectin and pyruvate dehydrogenase kinase $4(P d k 4)$ in adipocytes (Hogan \& Stephens 2005, Story \& Stephens 2006, White 
et al. 2007, 2016, Lin et al. 2014). Despite the identification of these direct STAT5 target genes, very little is known about the molecular mechanisms that contribute to the ability of STAT5A to regulate gene expression in adipocytes. To further investigate the functions of STAT5A in adipocytes, we sought to identify novel proteins that interact with STAT5A by performing a nonbiased co-immunoprecipitation and mass spectrometry approach (Richard et al. 2017). This approach identified DBC1 (deleted in breast cancer 1) as a potential STAT5Ainteracting protein.

DBC1, also referred to as CCAR2 (cell cycle and apoptosis regulator 2), is a pleiotropic protein that is primarily localized in the nucleus. DBC1 has been shown to physically interact and negatively regulate several epigenetic modifiers including sirtuin 1 (SIRT1), histone deacetylase 3 (HDAC3) and suppressor of variegation 3-9 homolog 1 (SUV39H1) (Zhao et al. 2008, Li et al. 2009, Chini et al. 2010). SIRT1 is a NAD-dependent deacetylase involved in a variety of cellular processes including regulation of obesity-associated metabolic diseases, cancer, aging and cellular senescence (Rahman \& Islam 2011). A loss of DBC1 expression is associated with increased SIRT1 activity in A459 human alveolar basal epithelial cells (Zhao et al. 2008). DBC1 also interacts and negatively regulates estrogen receptor $\beta$ (ER $\beta)$ and breast cancer 1 susceptibility protein 1 (BRCA1) (Hiraike et al. 2010, Koyama et al. 2010). In addition, DBC1 can be present in a complex with and positively regulate estrogen receptor $\alpha(\mathrm{ER} \alpha)$, androgen receptor (AR) and nuclear receptor subfamily 1 , group D, member 2 (Rev-erb $\alpha$ ) (Fu et al. 2009, Yu et al. 2011, Chini et al. 2013). DBC1 has also been shown to physically interact with and positively regulate IKK- $\alpha$ and IKK- $\beta$; two kinases that are part of the inhibitor of kappa B Kinase (IKK) complex that affects nuclear factor kappa B (NF-kB) signaling and transcriptional activity (Kong et al. 2015).

To date, most DBC1 studies have been performed in tumor cells and little is known about the function of DBC1 in adipocytes. However, studies have shown that knockdown of DBC1 in 3T3-L1 preadipocytes promotes adipocyte development in vitro (Moreno-Navarrete et al. 2015a). Also, DBC1-knockout mice have increased fat accumulation in adipose tissue, but remain metabolically healthy (Escande et al. 2015). DBC1-knockout mice maintained insulin sensitivity, had lower circulating free fatty acids and were protected against atherosclerosis and liver steatosis following diet-induced obesity (Escande et al. 2015). Other studies have shown that loss of DBC1 in 3T3-L1 adipocytes results in decreased expression of inflammatory markers such as interleukin 6 (IL-6), monocyte chemoattractant protein 1 (MCP1) and tumor necrosis factor-alpha $(\mathrm{TNF} \alpha)$, indicating that $\mathrm{DBC} 1$ may influence adipocyte inflammation (Moreno-Navarrete et al. 2015b). DBC1 has also been implicated in senescence of preadipocytes as loss of DBC1 protects again cellular senescence and senescence-driven inflammation in obesity (Escande et al. 2014).

Our studies are the first to show that DBC1 is present in a complex with STAT5A under physiological conditions in the nucleus of adipocytes. However, knockdown approaches revealed that $\mathrm{DBC} 1$ does not have a profound effect on the ability of GH to regulate STAT5 target genes. In studies to observe an impact of DBC1 loss in adipocytes, we found that DBC1 can impact TNF $\alpha$-mediated lipolysis in mature 3T3-L1 adipocytes. Although DBC1 did not have a profound effect on TNF $\alpha$-mediated changes in Mcp-1 or adiponectin expression, the loss of DBC1 resulted in increased Glut4 expression.

\section{Materials and methods}

\section{Cell culture}

Murine 3T3-L1 preadipocytes (obtained from Dr Howard Green at Harvard University) were grown in Dulbecco's Modified Eagle's Media (DMEM) (Sigma-Aldrich) with $10 \%$ bovine calf serum. Two days after confluence, the preadipocytes were induced to differentiate using a standard protocol and 3-isobutyl-methylxanthine, dexamethasone, insulin (MDI) induction cocktail plus $10 \%$ fetal bovine serum (FBS) in DMEM (Richard et al. 2017). HyClone calf and FBS were purchased from Thermo Scientific or GE Healthcare Life Sciences (Marlborough, $\mathrm{MA})$. The medium was changed every $48-72 \mathrm{~h}$ during growth and differentiation. Cells were serum deprived by changing the medium to DMEM containing $1 \%$ calf serum for $16-24 \mathrm{~h}$ before treatment with murine $\mathrm{GH}$ (mGH) or murine TNF $\alpha$. Recombinant murine GH was obtained from Dr. A.F. Parlow at the National Hormone and Peptide Program (NHPP; Torrance, CA). Recombinant murine TNF $\alpha$ was purchased from Thermo Fisher (Cat \#: PMC3013).

\section{siRNA-mediated knockdown}

3T3-L1 adipocytes (5-7 days post-MDI) were trypsinized and re-plated in 6-well, 12-well or 24-well plates at a density of $5.8 \times 10^{5}$ cells $/ \mathrm{cm}^{2}$ in antibiotic-free medium (10\% FBS/DMEM). Using the protocol from Dharmacon, adipocytes were transfected with $50 \mathrm{nM}$ siRNA (Dharmacon, Lafayette, CO, USA; Non-targeting siRNA Cat 
\#: D-001810-10-50, siRNA targeting DBC1 Cat \#: L-04783700-0005) and the DharmaFECT Duo reagent (Dharmacon, Cat \#: T-2010-03) in OptiMEM reduced serum medium (Thermo Fisher; Cat \#: 31985088). Non-targeting siRNA was used as negative control. After $24 \mathrm{~h}$, siRNA-containing media was removed, replaced with antibiotic-free 10\% FBS/DMEM and cells were transfected again with $50 \mathrm{nM}$ siRNA. After $24 \mathrm{~h}$, the media was removed and replaced with antibiotic-free 10\% FBS/DMEM. Cell monolayers were collected $72 \mathrm{~h}$ following the initial transfection and harvested for protein in immunoprecipitation (IP) buffer, and for RNA in buffer provided in the RNeasy mini kit (Qiagen) to assess knockdown efficiency. Knockdown of Cyclophilin B was used as a positive control to assess siRNA transfection efficiency in 3T3-L1 adipocytes because it is a well-characterized housekeeping gene that is not affected by experimental treatments in adipocytes and has been validated in our lab. (Dharmacon; Cat \#: D-001820-02-05).

\section{RNA analysis}

Adipocyte monolayers were harvested in lysis buffer from the RNeasy mini kit and total RNA was isolated from harvested cells using the RNeasy mini kit (Qiagen). Ten microliters of purified RNA were used for reverse transcription (RT) PCR to generate cDNA according to the Applied Biosystems protocol (Applied Biosystems; Cat \#: 4368813). cDNA was quantitated using the realtime quantitative PCR (qPCR) method in a total volume of $10 \mu \mathrm{L}(2 \mu \mathrm{L}$ DNA and $8 \mu \mathrm{L}$ reaction master mix) using an Applied Biosystems 7900HT Fast Real-Time PCR System and the qPCR amplification program specified in the kit (Clontech, Mountain View, CA; Cat \#: RR420A). Cyclophilin B (Ppib), Ubiquitin B ( $\mathrm{Ubb})$ and Nono were used as endogenous controls. The following mouse genes were examined by RT-qPCR: Ppib, Ubb, Nono, Dbc1 (Ccar2),
Socs3, Cish, Bcl6, Igf1, Mcp1, Glut4 and adiponectin (Adipoq) using primers purchased from Integrated DNA Technologies (IDT, Coralville, IA, USA). Primer sequences for each gene are shown in Table 1.

\section{Whole-cell extract preparation}

Adipocyte monolayers were rinsed once with PBS and then harvested in non-denaturing IP buffer as previously described (Richard et al. 2017). The cell extract was subjected to a freeze/thaw cycle at $-80^{\circ} \mathrm{C}$ and then passed through a 20-gauge needle three times. The whole-cell extract was further purified by centrifugation at $13,000 \boldsymbol{g}$ for $10 \mathrm{~min}$ at $4^{\circ} \mathrm{C}$

\section{Subcellular fractionation}

Mature 3T3-L1 adipocytes were treated with vehicle or murine $\mathrm{GH}$ prior to subcellular fractionation. Adipocyte monolayers from twenty $10 \mathrm{~cm}$ culture plates were scraped into nuclear homogenization buffer (NHB) $(20 \mathrm{mM}$ Tris $\mathrm{pH}$ 7.4, $10 \mathrm{mM} \mathrm{NaCl}$, and $3 \mathrm{~mL} \mathrm{MgCl}_{2}$ ) as previously described (Richard et al. 2017). After adding 0.15\% IGEPAL CA-630 (from 10\% stock) to the cell suspension, it was homogenized on ice using 16 strokes in a Dounce homogenizer. The extract was centrifuged at $517 \boldsymbol{g}$ in a Beckman GS-6KR centrifuge with a swinging bucket rotor. Subsequently, the supernatant containing cytosol and mitochondria was centrifuged as previously described (Richard et al. 2017). The nuclear pellet from the first centrifugation was washed once with half of the initial volume of NHB buffer and re-centrifuged at $4^{\circ} \mathrm{C}$ for $5 \mathrm{~min}$ at $57 \mathrm{~g}$ in the Beckman GS-6KR centrifuge. The supernatant was discarded, while the nuclear pellet was resuspended in IP buffer and incubated on ice for $1 \mathrm{~h}$. To break open the nuclei, the nuclear extract was passed through a $20 \mathrm{G}$ needle four times and further purified by centrifuging at $13,000 \mathrm{~g}$ for $10 \mathrm{~min}$ at $4^{\circ} \mathrm{C}$.

Table 1 Primer sequences for each gene examined by real-time qPCR.

\begin{tabular}{l} 
Gene \\
\hline Ppib \\
Ubb \\
Nono \\
Dbc1 (Ccar2) \\
Socs3 \\
Cish \\
Bcl6 \\
Igf1 \\
Mcp1 \\
Glut4 \\
Adiponectin
\end{tabular}

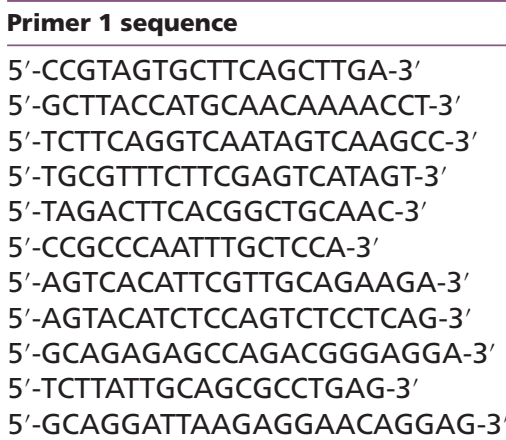

\begin{tabular}{l}
\hline Primer 2 sequence \\
\hline 5'-AGCAAGTTCCATCGTGTCATC-3' \\
5'-CCAGTGGGCAGTGATGG-3' \\
5'-CATCATCAGCATCACCACCA-3' \\
5'-CTTCCAGACATCCCACACAC-3' \\
5'-CGGGGAGCTAGTCCCGAA-3' \\
5'-GCTCCTTTCTCCTTCCATCC-3' \\
5'-CAGAGATGTGCCTCCATACTG-3' \\
5'-ATGCTCTTCAGTTCGTGTGT-3' \\
5'-TGGGGCGTTAACTGCATCTGG-3' \\
5'-GAGAATACAGCTAGGACCAGTG-3' \\
5'-TGTCTGTACGATTGTCAGTGG-3'
\end{tabular}




\section{Measurement of protein concentration}

Protein content of cell extracts was quantified using the Bicinchoninic acid (BCA) assay kit (Sigma-Aldrich; Cat \#: BCA1),

\section{Gel electrophoresis and immunoblotting}

Samples were separated on 7.5, 10 or 15\% SDS-PAGE (acrylamide; National Diagnostics, Atlanta, GA, USA; Cat \#: EC-890) and transferred to nitrocellulose membranes (BioRad; Cat \#: 162-0115) in $25 \mathrm{mM}$ Tris, $192 \mathrm{mM}$ glycine and 20\% methanol. After the transfer, the membrane was blocked in $4 \%$ milk for $1 \mathrm{~h}$ at room temperature and then immunoblotted. Results were visualized with horseradish peroxidase-conjugated secondary antibodies (Jackson ImmunoResearch) and enhanced chemiluminescence (Pierce/Thermo Scientific).

\section{Immunoprecipitation (IP)}

Cell extracts (300-400 $\mu$ g total protein) were incubated with $4-5 \mu \mathrm{g}$ of immunoprecipitating antibody, diluted in IP buffer, overnight on a mini-tube rotator at $4^{\circ} \mathrm{C}$. Protein A-conjugated agarose beads (IPA300 Protein A Resin; Repligen, Waltham, MA, USA; Cat \#: 10-2003-02) were added to the antibody-epitope mixture, and the conjugation reaction proceeded for an additional 3-4h at $4^{\circ} \mathrm{C}$ with rotation. Following conjugation to the bead resin, the beads were pelleted by centrifugation at 16,750 $\boldsymbol{g}$ for $3 \mathrm{~min}$ at $4^{\circ} \mathrm{C}$. The supernatant was removed by aspiration and the beads were washed three times with ice cold 1X IP buffer. Between each wash, the beads were pelleted by centrifugation at $13,000 \mathrm{~g}$ for $3 \mathrm{~min}$ at $4^{\circ} \mathrm{C}$ and the supernatant was removed by aspiration. After the final wash, the IP antibody and immunoprecipitated proteins were eluted from the bead resin into $2 \times$ SDS loading buffer by boiling the samples for $10 \mathrm{~min}$ at $100^{\circ} \mathrm{C}$. Samples were flicked every $2 \mathrm{~min}$ during heat step to ensure efficient elution. The samples were briefly centrifuged and the supernatants were analyzed by SDS-PAGE and immunoblotting. A mock sample containing only IP antibody and IP buffer (no cell extract) was used as a negative control for each IP experiment.

\section{SIRT1 activity measurement}

The deacetylase activity of SIRT1 was measured using a fluorometric assay kit (Enzo Life Sciences, Farmingdale, NY, USA; Cat \#: BML-AK500-0001). Cell extracts (75 $\mu \mathrm{g}$ total protein) were incubated with $5 \mu \mathrm{g}$ of anti-SIRT1 antibody using the immunoprecipitation method described earlier. After the final wash, the samples were resuspended in $100 \mu \mathrm{L}$ of assay buffer provided by the assay kit. The manufacturer's instructions were followed and optimized for our samples. Briefly, the deacetylase reaction was initiated by adding $50 \mu \mathrm{M}$ of Fluor de lys substrate and $1 \mathrm{mM} \mathrm{NAD+}$ (SIRT1 substrate) to samples. After incubating the samples for $20 \mathrm{~min}$ at room temperature, the reactions were stopped by addition of $1 \mathrm{X}$ Fluor de lys developer that contained either $1 \mu \mathrm{M}$ trichostatin A (TricA; HDAC inhibitor) or $2 \mathrm{mM}$ of nicotinamide (NAM; SIRT1 inhibitor). Samples were read in a fluorometer (SpectraMax M5) at an excitation wavelength of $360 \mathrm{~nm}$ and emission wavelength of $460 \mathrm{~nm}$.

\section{Measurement of glycerol and free fatty acid release}

3T3-L1 adipocytes were transfected with either nontargeting or DBC1 siRNA as described above. Fifty-four hours later, media was changed to 1\% calf/DMEM and cells were treated with equivalent volume of vehicle $(0.1 \%$ BSA/PBS) or $0.75 \mathrm{nM}$ TNF $\alpha$ overnight. After $16 \mathrm{~h}, 1 \%$ calf/DMEM was removed and replaced with incubation media that consisted of $2 \%$ fatty acid free bovine serum albumin (BSA; Sigma-Aldrich; Cat \#: A6003) and $0.1 \%$ glucose in glucose-free phenol red-free DMEM, and the cells were treated again with vehicle (0.1\% BSA/PBS) or $0.75 \mathrm{nM} \mathrm{TNF} \alpha$. After $2 \mathrm{~h}, 500 \mu \mathrm{L}$ of conditioned media was collected and stored at $-20^{\circ} \mathrm{C}$. Glycerol release was measured using $50 \mu \mathrm{L}$ of each sample according to the kit protocol (Millipore; Cat \#: OB100). Free fatty acid release was measured using $50 \mu \mathrm{L}$ of each sample according to the kit protocol (BioVision, Milpitas, CA, USA; Cat \#: K612).

\section{Antibodies}

Anti-STAT5A (L-20; sc1081; rabbit polyclonal) and anti-ERK1/2 (C-16; sc-93; rabbit polyclonal) antibodies were purchased from Santa Cruz Biotechnology. We used both mouse monoclonal (clone 8-5-2; 05-495) and rabbit polyclonal (07-586) anti-phospho-STAT5 A/B (Tyr 694/699) antibodies from Millipore to detect tyrosine phosphorylated STAT5 (STAT5pY). Antiadiponectin (PA1-054; rabbit polyclonal) antibody was purchased from Thermo Scientific. Anti-DBC1 (5693; rabbit polyclonal) and anti-SIRT1 (1F3; 8469; mouse monoclonal) antibodies were purchased from Cell Signaling Technology. 


\section{Statistical analysis}

All data were analyzed by two-tailed unpaired Student's t-test (using GraphPad Prism 7). Results from studies of cultured adipocytes are shown as mean \pm standard error of the mean (S.E.M.). Results were considered statistically significant when $P<0.05$.

\section{Results}

We previously utilized a semi-nonbiased screening approach to identify novel STAT5 interaction partners using co-immunoprecipitation and mass spectrometry. Pyruvate dehydrogenase complex-E2 (PDC-E2) was the first interacting protein that we reported from this screen (Richard et al. 2017). In our present study, we report that DBC1 was also identified as a potential novel STAT5A-interacting protein in 3T3-L1 adipocytes. To validate our mass spectrometry results, we performed co-immunoprecipitation with a STAT5A antibody followed by Western blotting using an anti-DBC1 antibody, and we examined both cytosolic and nuclear extracts from mature 3T3-L1 adipocytes in the absence or presence of an acute GH treatment, a condition that activates STAT5A (Fig. 1A). These studies revealed that DBC1 interacts with STAT5A in the nucleus and that the nuclear localization of DBC1, unlike STAT5A, was not dependent on GH-induced STAT5 tyrosine phosphorylation (Fig. 1A). To further validate that these two proteins were present in the same protein complex in adipocytes, we demonstrated that STAT5A could also be pulled down by a reverse co-immunoprecipitation using a DBC1 antibody (Fig. 1B). Together, these experiments corroborate that DBC1 is present in a complex with STAT5A under physiological conditions in the nucleus of adipocytes and that this interaction is not dependent upon GH stimulation or STAT5 tyrosine phosphorylation.

To assess the function of the DBC1/STAT5A interaction, we performed siRNA experiments to knockdown DBC1 in mature adipocytes. The goal was to determine if DBC1 expression had any effect on STAT5 phosphoactivation or transcriptional activity. In these studies, we examined the ability of GH to induce STAT5A activation by measuring STAT5A tyrosine phosphorylation. Western blot analysis showed that loss of DBC1 levels did not have an effect on levels of STAT5A or STAT5pY (Fig. 2).

To confirm that the loss of DBC1 protein affected its activity in adipocytes, we performed additional experiments to measure SIRT1 activity. It is well established that SIRT1 activity is negatively regulated by DBC1 (Kim
A

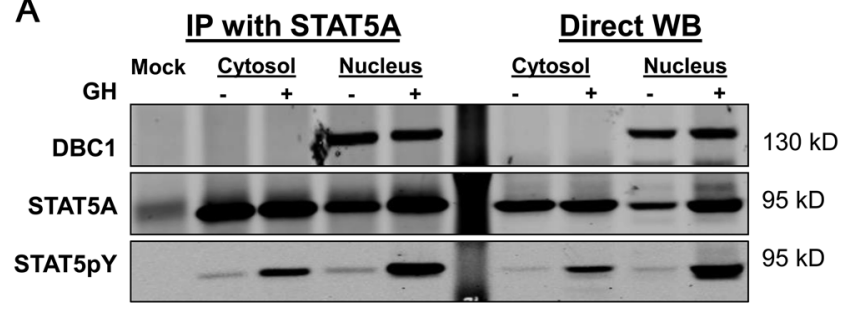

B

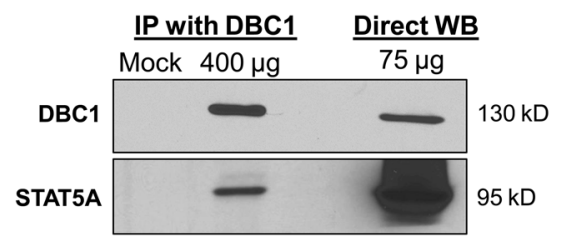

Figure 1

DBC1 is present in the nucleus of adipocytes and can be co-immunoprecipitated with STAT5A. (A) Fully differentiated 3T3-L1 adipocytes were treated with $5 \mathrm{nM}$ murine growth hormone $(\mathrm{GH})$ or an equivalent volume of vehicle $\left(10 \mathrm{mM} \mathrm{NaHCO}_{3}\right)$ for $20 \mathrm{~min}$. Monolayers were collected and subjected to subcellular fractionation. As shown in the left-hand portion of the figure, cytosolic and nuclear protein extracts $(300 \mu \mathrm{g}$ total protein/sample) were immunoprecipitated (IP) with an anti-STAT5A antibody. The mock sample contained anti-STAT5A antibody without cell extract. The right-hand portion of the figure are direct Western blot (WB) controls containing $75 \mu \mathrm{g}$ total protein that were directly subjected to Western blotting without IP. STAT5pY represents phosphorylated STAT5 at tyrosine 694/699. This is a representative figure of an experiment independently performed three times. (B) The monolayers of fully differentiated 3T3-L1 adipocytes were harvested and whole-cell protein extracts were prepared. The left-hand portion of the figure shows IP of whole-cell extract containing $400 \mu \mathrm{g}$ of total protein, anti-DBC1 antibody and IP buffer. Mock sample contained anti-DBC1 antibody without cell extract. The right-hand portion of the figure contains WB controls. This is a representative figure of an experiment independently performed three times on different groups of adipocytes.

et al. 2008, Zhao et al. 2008). As expected, our experiments showed an increase in SIRT1 activity when DBC1 protein levels were reduced $\left({ }^{* * *} P<0.001\right)$, suggesting that the knockdown of DBC1 also affected its activity in adipocytes as judged by SIRT1 activity (Fig. 3A). Induction of SIRT1 deacetylase activity in HeLa extract with the addition of $\mathrm{NAD}+$ or trichostatin A (TricA) was used as a control to confirm validity of the deacetylase assay (Fig. 3B).

Tyrosine phosphorylated STAT5A can activate or repress the expression of many genes. To determine if DBC1 expression had any effect on the transcriptional activity of STAT5, we examined the expression of several STAT5 target genes following GH treatment of mature adipocytes for 1-4h under control or DBC1-knockdown conditions. The expression of STAT5A target genes (Socs3, Cish and Bcl6) was assessed by qPCR. It is known that Socs 3 and Cish gene expression are highly induced $1 \mathrm{~h}$ following STAT5 activation (Matsumoto et al. 1997, Karlsson et al. 1999, Barclay et al. 2007), whereas Bcl6 gene 


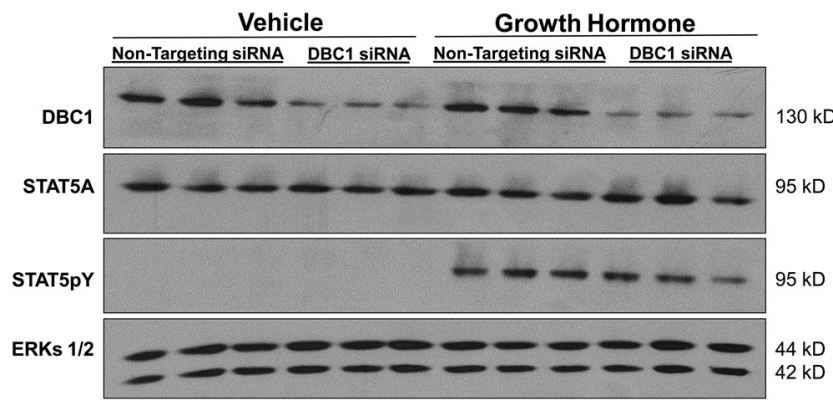

Figure 2

Knockdown of DBC1 does not affect STAT5A protein expression or STAT5 tyrosine phosphorylation in 3T3-L1 adipocytes. Fully differentiated 3T3-L1 adipocytes were transfected once per day for 2 days with non-targeting siRNA or DBC1 siRNA. After $48 \mathrm{~h}$, adipocytes were serum deprived for $4 \mathrm{~h}$ and treated with $5 \mathrm{nM}$ murine growth hormone $(\mathrm{GH})$ or equivalent volume of vehicle $\left(10 \mathrm{mM} \mathrm{NaHCO}_{3}\right)$ for $20 \mathrm{~min}$. Monolayers were collected and $75 \mu \mathrm{g}$ of total protein was separated using SDS-PAGE. Protein expression was visualized using Western blotting with the antibodies indicated to the left of panel. ERK was used as a loading control. Three biological replicates were used for each treatment group in each individual experiment. This is a representative figure of an experiment independently performed three times on different groups of adipocytes.

expression is reduced following $4 \mathrm{~h}$ of $\mathrm{GH}$ treatment (Lin et al. 2014). The data showed the expected regulation of Socs3, Cish and Bcl6 gene expression following $1 \mathrm{~h}$ of $\mathrm{GH}$ treatment. However, there were no differences in mRNA levels of these genes in adipocytes with reduced DBC1 expression (Fig. 4). Similar results were observed for other STAT5A target genes such as adiponectin, Fasn and Pdk4 (data not shown).

Although DBC1 did not have a profound impact on STAT5A transcriptional activity, there is evidence in the literature that DBC1 may affect adipose tissue inflammation (Moreno-Navarrete et al. 2015b). We explored this on the cellular level by investigating the ability of DBC1 to modulate TNF $\alpha$ action in adipocytes. $\mathrm{TNF} \alpha$ is known to increase basal lipolysis, promote the expression and secretion of pro-inflammatory cytokines, such as IL-6, and reduce the secretion of anti-inflammatory hormones, such as adiponectin (Berghe et al. 2000, He et al. 2016). To investigate the effects of $\mathrm{DBC} 1$ on TNFa action, we used siRNA to knockdown DBC1 in mature 3T3-L1 adipocytes. Gene expression analysis showed that a loss of DBC1, independent of TNF $\alpha$ treatment, increased Glut4 gene expression $\left({ }^{* * *} P<0.01\right.$ for Vehicle treatment and ${ }^{* *} P<0.01$ for TNF $\alpha$ treatment) but did not have a profound effect on TNF $\alpha$-mediated changes in expression of $M c p 1$ or adiponectin (Fig. 5A). Western blot analysis was used to assess the level of DBC1 protein knockdown and the effectiveness of TNF $\alpha$ treatment, as judged by reduced adiponectin protein levels (Fig. 5B), which was consistent

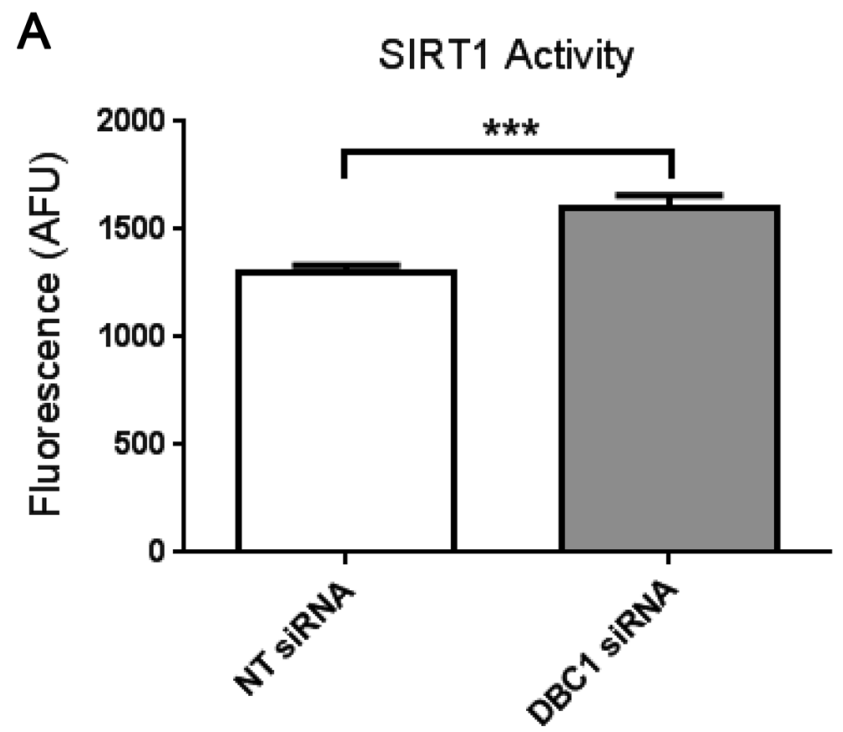

B Assay Controls

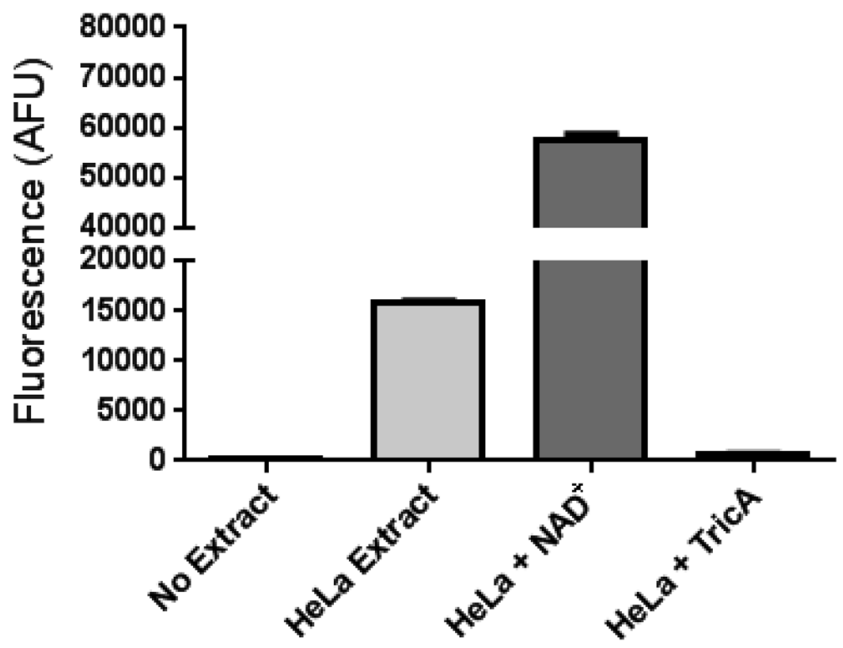

Figure 3

Knockdown of DBC1 increases the deacetylase activity of SIRT1 in 3T3-L1 adipocytes. (A) SIRT1 deacetylase activity was measured in samples prepared by immunoprecipitating SIRT1 from 3T3-L1 adipocytes transfected with non-targeting (NT) or DBC1 siRNA. Each IP reaction contained $75 \mu \mathrm{g}$ of total protein, anti-SIRT1 antibody and IP buffer. (B) HeLa extract was used as a control to assess SIRT1 deacetylase activity. The graph shows the requirement of NAD+ for SIRT deacetylase activity and Trichostatin A (TricA) inhibiting the Fluor de Lys substrate deacetylation in HeLa nuclear extract. Statistical significance was determined using a Student's $t$-test and assigned as $* * * P<0.001$.

with the changes observed in adiponectin gene expression (Fig. 5A). We also assessed whether DBC1 expression had an impact on TNF $\alpha$-mediated lipolysis. Both free fatty acid and glycerol release were measured from mature adipocytes following TNF $\alpha$ treatment in control and DBC1-knockdown adipocytes. We observed a modest, but 

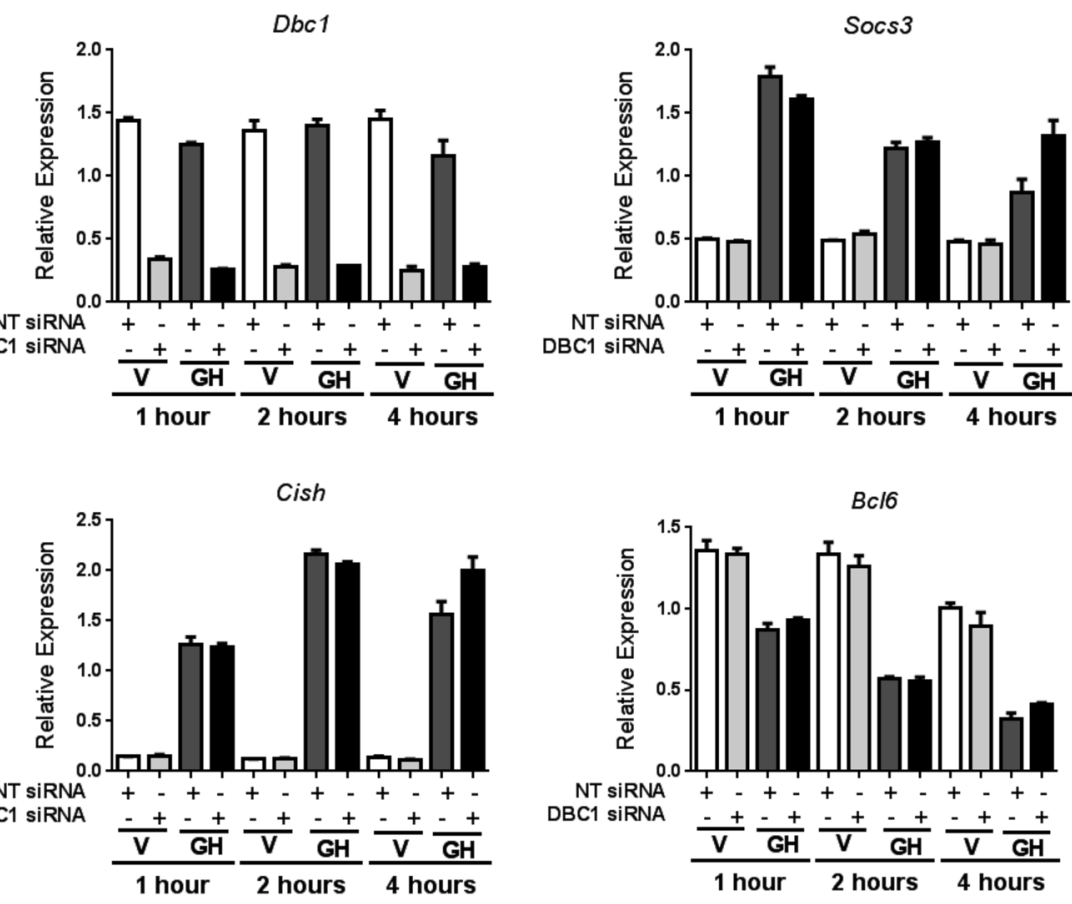

statistically significant, decrease in TNF $\alpha$-induced glycerol $\left({ }^{* * *} P<0.005\right)$ and free fatty acid $\left({ }^{\star} P<0.05\right)$ release with reduced DBC1 expression (Fig. 6).

\section{Discussion}

STAT5A is a transcription factor that can mediate the effects of GH (Zvonic et al. 2003) and promote adipocyte development (Floyd \& Stephens 2003, Stewart et al. 2011). In adipocytes, STAT5A regulates genes that contribute to insulin sensitivity and the endocrine properties of adipocytes (Hogan \& Stephens 2005, White et al. 2007, 2016). To further understand the mechanisms involved in the contribution of STAT5A to fat cell function, we sought to identify novel STAT5A-interacting proteins in adipocytes using a co-immunoprecipitation/mass spectrometry approach. We recently published data describing this approach and the identification of components of the pyruvate dehydrogenase complex as STAT5A-binding proteins (Richard et al. 2017). Another STAT5A-binding protein that we identified with this approach was the nuclear protein, DBC1. DBC1 has been shown to mediate cellular responses to stress and regulate the activity of a variety of enzymes and transcription factors in different cell types (Joshi et al. 2013). Our novel observations demonstrate that DBC1 can be present in the same protein complex as STAT5A in the nucleus of 3T3-L1 adipocytes (Fig. 1). This interaction was observed with endogenous proteins and could be detected by

\begin{abstract}
Figure 4
Knockdown of DBC1 in mature adipocytes does not affect the ability of $\mathrm{GH}$ to alter the expression of STAT5A target genes. Fully differentiated 3T3-L1 adipocytes were transfected once per day for two days with non-targeting (NT) siRNA or DBC1 siRNA. After $48 \mathrm{~h}$, adipocytes were serum deprived for $4 \mathrm{~h}$ and treated with $5 \mathrm{nM}$ murine growth hormone $(\mathrm{GH})$ or equivalent volume of vehicle $(\mathrm{V} ; 10 \mathrm{mM} \mathrm{NaHCO}$ ) for 1,2 , or $4 \mathrm{~h}$. Total RNA was isolated and subjected to quantitative RT-PCR analysis. Each graph represents a different gene. The examined genes were normalized to Nono. This is a representative figure of an experiment independently performed three times on different groups of adipocytes.
\end{abstract}

immunoprecipitating either STAT5A or DBC1. In addition to this observation, our results reveal that this interaction is not dependent on STAT5A tyrosine phosphorylation (Fig. 1A).

To assess the potential function of the STAT5A/DBC1 association, we examined STAT5A expression levels as well as STAT5A tyrosine phosphorylation in adipocytes that had substantially decreased levels of DBC1. These studies revealed that loss of DBC1 did not alter STAT5A total protein levels or its ability to be activated, as judged by tyrosine phosphorylation (Fig. 2). We also examined the ability of STAT5A to regulate gene expression in adipocytes with reduced DBC1 expression. To our surprise, there were no profound changes in the expression of several STAT5A target genes in DBC1-deficient adipocytes, which strongly indicates that DBC1 levels do not affect the transcriptional activity of STAT5A (Fig. 4). Although there were some modest differences in Socs3 and Cish gene expression following 4-h $\mathrm{GH}$ treatment in DBC1 knockdown adipocytes, these results were not statistically significant. The effectiveness of siRNA-mediated DBC1 knockdown was assessed by examining gene and protein expression levels of DBC1 (Figs 2, 4 and 5) as well as SIRT1 deacetylase activity (Fig. 3) since DBC1 is a well-known negative regulator of SIRT1 (Kim et al. 2008, Zhao et al. 2008, Liu et al. 2016). As expected, we observed an increase in SIRT1 activity, indicating that the activity of DBC1 was likely altered in the DBC1-knockdown adipocytes (Fig. 3). Presumably, we only observed a modest increase in SIRT1 
A
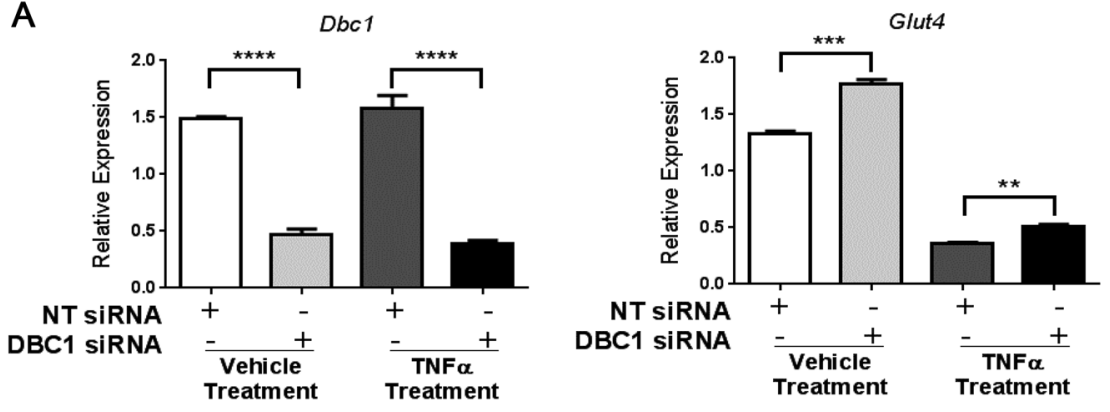

\section{Figure $\mathbf{5}$}

Knockdown of DBC1 in mature adipocytes results in increased Glut4 gene expression but does not alter TNF $\alpha$-mediated changes in gene expression. Fully differentiated 3T3-L1 adipocytes were transfected once per day for two days with non-targeting (NT) siRNA or DBC1 siRNA. After $54 \mathrm{~h}$, media was replaced with $1 \%$ calf and cells were treated with $0.75 \mathrm{nM}$ TNF $\alpha$ overnight or equivalent volume of vehicle (0.1\% BSA/PBS). After $16 \mathrm{~h}$, cells were re-treated again with vehicle or TNF $\alpha$ for $2 \mathrm{~h}$. Finally, monolayers were harvested for RNA and protein. (A) Total RNA was isolated and subjected to quantitative RT-PCR analysis. The examined genes (each represented by separate graph) were normalized to Nono. Statistical significance was determined using a Student's $t$-test and assigned as **P<0.01, $* * * P<0.001$, and $* * * * P<0.0001$. (B) $75 \mu \mathrm{g}$ of total protein was separated using SDS-PAGE. Protein expression was visualized using Western blotting and the antibodies indicated on the left of panel. Three biological replicates were used for each treatment group in each individual experiment. This is a representative figure of an experiment independently performed three times on different groups of adipocytes.

activity because DBC1 is only one of many regulators of SIRT1 activity in mature adipocytes. Other studies have shown that a variety of other factors including the cAMP/ PKA pathway and AROS (active regulator of SIRT1) can positively regulate SIRT1 (Kim et al. 2007, Chao \& Tontonoz 2012). Collectively, our observations suggest that DBC1 levels do not affect the transcriptional activity of STAT5A.

Since there was evidence suggesting that DBC1 may have a pro-inflammatory role in adipocytes by increasing the expression of NF-кB-regulated inflammatory cytokines (Moreno-Navarrete et al. 2015b), we examined the actions of TNF $\alpha$ on adipocytes with reduced levels of DBC1. TNF $\alpha$ can induce insulin resistance, in part, through the transcriptional repression of the Glut4 gene in adipocytes (Stephens \& Pekala 1991, Stephens et al. 1997, Ruan et al. 2002a) and by activating NF-кB (Ruan et al. $2002 b$ ). In addition, TNF $\alpha$ can repress adiponectin gene expression in mouse and human adipocytes (Fasshauer et al. 2002, Degawa-Yamauchi et al. 2005). Both increased GLUT4 protein expression in adipocytes (Carvalho et al. 2005, Atkinson et al. 2013) and increased adiponectin levels (Fu et al. 2005) are associated with improvements in insulin sensitivity. Knockdown of DBC1 did not alter TNFo-mediated induction of pro-inflammatory MCP-1 or repression of anti-inflammatory adiponectin (Fig. 5A). However, we consistently observed that a loss of DBC1 protein resulted in increased Glut4 gene expression, which suggests a potential role for DBC1 in glucose regulation. Studies in HepG2 cells have shown a knockdown of DBC1 results in the upregulation of PEPCK (phosphoenolpyruvate carboxykinase) and consequently effects glucose production (Nin et al. 2014). Experiments performed with inguinal fat tissue from DBC1-null mice also showed increased PEPCK expression in DBC1deficient tissue (Moreno-Navarrete et al. 2015b). Although additional experiments will be required to determine if there are any direct effects of DBC1 on glucose metabolism or Glut4 gene expression, our results suggest that DBC1 is a likely a negative regulator of GLUT4 in adipocytes (Fig. 5A).

Although the loss of DBC1 did not profoundly affect $\mathrm{TNF} \alpha$ regulation of several genes in adipocytes (Fig. 5), we did observe that alteration of DBC1 levels affected TNFo-induced lipolysis. Our studies revealed a modest 

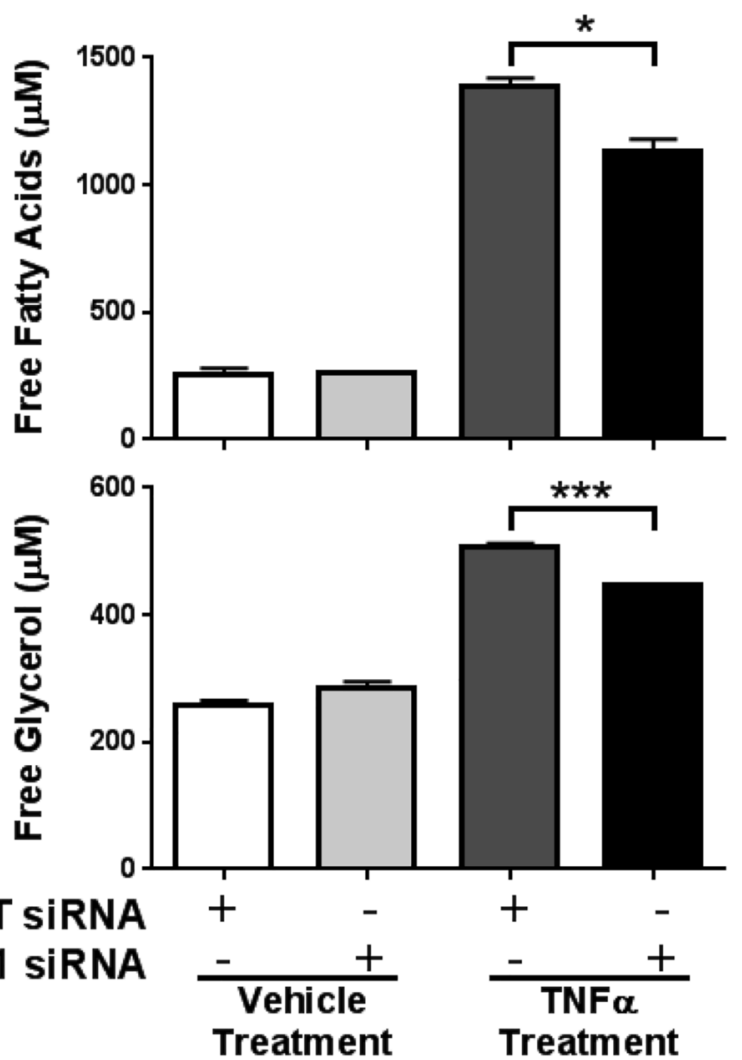

\section{Figure 6}

Knockdown of DBC1 in mature adipocytes modestly suppresses TNF $\alpha$-induced lipolysis. Fully differentiated 3T3-L1 adipocytes were transfected once per day for two days with non-targeting (NT) siRNA or DBC1 siRNA. After $54 \mathrm{~h}$, media was replaced with incubation media and cells were treated with $0.75 \mathrm{nM}$ TNF $\alpha$ or equivalent volume of vehicle (0.1\% BSA/PBS) overnight. After $12 \mathrm{~h}$, cells were treated again with vehicle or TNF $\alpha$ for $2 \mathrm{~h}$. fifty microliters of conditioned medium was used for free fatty acid assay and glycerol assay. Statistical significance was determined using a Student's $t$-test and assigned as $* P<0.05$ and $* * * P<0.005$. This is a representative figure of an experiment independently performed two times on different groups of adipocytes.

but significant reduction in glycerol and free fatty acid release in DBC1-knockdown adipocytes under TNF $\alpha$ stimulated conditions (Fig. 6). This effect could be considered metabolically favorable, which is also evident by the increased Glut4 expression we observed with loss of DBC1. Overall, our observations are consistent with the phenotype of the DBC1-knockout mice fed a high-fat diet that are more insulin sensitive and have decreased circulating FFA (Escande et al. 2015). Our data showing that loss of DBC1 increases Glut4 expression and reduces TNFo-induced lipolysis suggests that the loss of DBC1 in adipocytes may contribute to the metabolically protected phenotype of the DBC1-null mice. Although additional experiments in adipocyte-specific DBC1 knockout mice will be needed, the current data are consistent with a role of DBC1 in promoting metabolic dysfunction. Decreased FFA levels are typically associated with a metabolically healthy phenotype. Whereas an increase in glycerol and fatty acids is often harmful and associated with the clinical manifestations of metabolic syndrome, which also include obesity and insulin resistance (Boden 1999, Arner \& Rydén 2015). Our results show a decrease in glycerol and free fatty acid release from TNF $\alpha$-stimulated DBC1deficient adipocytes that occurs in a cell autonomous manner and suggest that DBC1 plays a direct or indirect role in the ability of TNF $\alpha$ to induce lipolysis.

Although these novel observations revealed that DBC1 is present in a protein complex with STAT5A in adipocyte nuclei (Fig. 1), most of our functional studies reproducibly generated negative observations. The loss of DBC1 did not affect STAT5A expression (Fig. 2), STAT5A tyrosine phosphorylation (Fig. 2) or the expression of STAT5A target genes (Fig. 4). Although DBC1 is clearly in a complex with STAT5, and loss of DBC1 did result in expected increase in SIRT1 activity (Fig. 3), we were unable to perturb STAT5A expression, activation or activity. We did, however, have two consistent effects that accompanied loss of DBC1 expression in adipocytes: (1) increased Glut4 expression and (2) a statistically significant decrease in TNF $\alpha$-induced lipolysis. Nevertheless, it is unlikely that these functions of DBC1 are related to its interaction with STAT5A. Further studies are needed to identify additional proteins present in the DBC1/STAT5A complex, to elucidate the function of the DBC1/STAT5A interaction and to investigate the mechanism of DBC1's ability to modulate TNF $\alpha$-induced lipolysis.

Declaration of interest

The authors declare that there is no conflict of interest that could be perceived as prejudicing the impartiality of the research reported.

\section{Funding}

This work was supported by National Institutes of Health Grant R01DK052968 to J M S.

\section{Acknowledgements}

This project used Genomics Core facilities that are supported in part by COBRE (NIH8 1P30GM118430-02) and NORC (NIH 2P30DK072476) center grants from the National Institutes of Health.

\section{References}

Arner P \& Rydén M 2015 Fatty acids, obesity and insulin resistance. Obesity Facts 8 147-155. (https://doi.org/10.1159/000381224) 
Atkinson BJ, Griesel BA, King CD, Josey MA \& Olson AL 2013 Moderate GLUT4 overexpression improves insulin sensitivity and fasting triglyceridemia in high-fat diet-fed transgenic mice. Diabetes 62 2249-2258. (https://doi.org/10.2337/db12-1146)

Barclay JL, Anderson ST, Waters MJ \& Curlewis JD 2007 Regulation of suppressor of cytokine signaling 3 (SOC3) by Growth Hormone in pro-B cells. Molecular Endocrinology 21 2503-2515. (https://doi. org/10.1210/me.2006-0498)

Berghe W, Vermeulen L, De Wilde G, De Bosscher K, Boone E \& Haegeman G 2000 Signal transduction by tumor necrosis factor and gene regulation of the inflammatory cytokine interleukin-6. Biochemical Pharmacology 60 1185-1195. (https://doi.org/10.1016/ S0006-2952(00)00412-3)

Boden G 1999 Free fatty acids, insulin resistance, and type 2 diabetes mellitus. Proceedings of the Association of American Physicians 111 241-248. (https://doi.org/10.1046/j.1525-1381.1999.99220.x)

Carvalho E, Kotani K, Peroni OD \& Kahn BB 2005 Adipose-specific overexpression of GLUT4 reverses insulin resistance and diabetes in mice lacking GLUT4 selectively in muscle. American Journal of Physiology: Endocrinology and Metabolism 289 E551-E561. (https://doi. org/10.1152/ajpendo.00116.2005)

Chao LC \& Tontonoz P 2012 SIRT1 regulation - it ain't all NAD. Molecular Cell 45 9-11. (https://doi.org/10.1016/j. molcel.2011.12.017)

Chini CCS, Escande C, Nin V \& Chini EN 2010 HDAC3 is negatively regulated by the nuclear protein DBC1. Journal of Biological Chemistry 285 40830-40837. (https://doi.org/10.1074/jbc.M110.153270)

Chini CCS, Escande C, Nin V \& Chini EN 2013 DBC1 (Deleted in Breast Cancer 1) modulates the stability and function of the nuclear receptor Rev-erbo. Biochemical Journal 451 453-461. (https://doi. org/10.1042/BJ20121085)

Degawa-Yamauchi M, Moss KA, Bovenkerk JE, Shankar SS, Morrison CL, Lelliott CJ, Vidal-Puig A, Jones R \& Considine RV 2005 Regulation of adiponectin expression in human adipocytes: effects of Adiposity, glucocorticoids, and tumor necrosis factor $\alpha$. Obesity Research 13 662-669. (https://doi.org/10.1038/oby.2005.74)

Escande C, Nin V, Pirtskhalava T, Chini CC, Thereza Barbosa M, Mathison A, Urrutia R, Tchkonia T, Kirkland JL \& Chini EN 2014 Deleted in breast cancer 1 regulates cellular senescence during obesity. Aging Cell 13 951-953. (https://doi.org/10.1111/acel.12235)

Escande C, Nin V, Pirtskhalava T, Chini CCS, Tchkonia T, Kirkland JL \& Chini EN 2015 Deleted in breast cancer 1 limits adipose tissue fat accumulation and plays a key role in the development of metabolic syndrome phenotype. Diabetes 64 12-22. (https://doi.org/10.2337/ db14-0192)

Fasshauer M, Klein J, Neumann S, Eszlinger M \& Paschke R 2002 Hormonal regulation of adiponectin gene expression in 3T3-L1 adipocytes. Biochemical and Biophysical Research Communications 290 1084-1089. (https://doi.org/10.1006/bbrc.2001.6307)

Floyd ZE \& Stephens JM 2003 STAT5A promotes adipogenesis in nonprecursor cells and associates with the glucocorticoid receptor during adipocyte differentiation. Diabetes 52 308-314. (https://doi. org/10.2337/diabetes.52.2.308)

Fu Y, Luo N, Klein RL \& Garvey WT 2005 Adiponectin promotes adipocyte differentiation, insulin sensitivity, and lipid accumulation Journal of Lipid Research 46 1369-1379. (https://doi.org/10.1194/jlr. M400373-JLR200)

Fu J, Jiang J, Li J, Wang S, Shi G, Feng Q, White E, Qin J \& Wong J 2009 Deleted in breast cancer 1, a novel androgen receptor (AR) coactivator that promotes AR DNA-binding activity. Journal of Biological Chemistry 284 6832-6840 (https://doi.org/10.1074/jbc. M808988200)

He Y, Lu L, Wei X, Jin D, Qian T, Yu A, Sun J, Cui J \& Yang Z 2016 The multimerization and secretion of adiponectin are regulated by TNF-alpha. Endocrine 51 456-468. (https://doi.org/10.1007/s12020015-0741-4)
Hiraike H, Wada-Hiraike O, Nakagawa S, Koyama S, Miyamoto Y, Sone K, Tanikawa M, Tsuruga T, Nagasaka K, Matsumoto Y, et al. 2010 Identification of DBC1 as a transcriptional repressor for BRCA1. British Journal of Cancer 102 1061-1067. (https://doi.org/10.1038/sj. bjc.6605577)

Hogan JC \& Stephens JM 2005 The regulation of fatty acid synthase by STAT5A. Diabetes 54 1968-1975. (https://doi.org/10.2337/ DIABETES.54.7.1968)

Joshi P, Quach OL, Giguere SSB \& Cristea IM 2013 A functional proteomics perspective of DBC1 as a regulator of transcription. Journal of Proteomics and Bioinformatics Supplement 2 1-8. (https;//doi. org/10.4172/jpb.S2-002)

Karlsson H, Gustafsson J-Å \& Mode A 1999 Cis desensitizes GH induced Stat5 signaling in rat liver cells. Molecular and Cellular Endocrinology 154 37-43. (https://doi.org/10.1016/S0303-7207(99)00101-X)

Kim E-J, Kho J-H, Kang M-R \& Um S-J 2007 Active regulator of SIRT1 cooperates with SIRT1 and facilitates suppression of p53 activity. Molecular Cell 28 277-290. (https://doi.org/10.1016/j. molcel.2007.08.030)

Kim J-E, Chen J \& Lou Z 2008 DBC1 is a negative regulator of SIRT1. Nature 451 583-586. (https://doi.org/10.1038/nature06500)

Kong S, Dong H, Song J, Thiruppathi M, Prabhakar BS, Qiu Q, Lin Z, Chini E, Zhang B \& Fang D 2015 Deleted in breast cancer 1 suppresses B cell activation through RelB and is regulated by IKKo phosphorylation. Journal of Immunology 3685-3693. (https://doi. org/10.4049/jimmunol.1500713)

Koyama S, Wada-Hiraike O, Nakagawa S, Tanikawa M, Hiraike H, Miyamoto Y, Sone K, Oda K, Fukuhara H, Nakagawa K, et al. 2010 Repression of estrogen receptor $\beta$ function by putative tumor suppressor DBC1. Biochemical and Biophysical Research Communications 392 357-362. (https://doi.org/10.1016/j. bbrc.2010.01.025)

Li Z, Chen L, Kabra N, Wang C, Fang J \& Chen J 2009 Inhibition of SUV39H1 methyltransferase activity by DBC1. Journal of Biological Chemistry 284 10361-10366. (https://doi.org/10.1074/jbc. M900956200)

Lin G, LaPensee CR, Qin ZS \& Schwartz J 2014 Reciprocal occupancy of BCL6 and STAT5 on Growth Hormone target genes: contrasting transcriptional outcomes and promoter-specific roles of p300 and HDAC3. Molecular and Cellular Endocrinology 395 19-31. (https://doi. org/10.1016/j.mce.2014.07.020)

Liu X, Ehmed E, Li B, Dou J, Qiao X, Jiang W, Yang X, Qiao S \& Wu Y 2016 Breast cancer metastasis suppressor 1 modulates SIRT1dependent p53 deacetylation through interacting with DBC1. American Journal of Cancer Research 6 1441-1449.

Matsumoto A, Masuhara M, Mitsui K, Yokouchi M, Ohtsubo M, Misawa H, Miyajima A \& Yoshimura A 1997 CIS, a cytokine inducible SH2 protein, is a target of the JAK-STAT5 pathway and modulates STAT5 activation. Blood 89 3148-3154.

Moreno-Navarrete JM, Moreno M, Vidal M, Ortega F, Serrano M, Xifra G, Ricart W \& Fernández-Real JM 2015a Deleted in breast cancer 1 plays a functional role in adipocyte differentiation. American Journal of Physiology: Endocrinology and Metabolism 308 E554-E561. (https://doi.org/10.1152/ajpendo.00286.2014)

Moreno-Navarrete JM, Moreno M, Vidal M, Ortega F, Ricart W \& Fernández-Real JM 2015b DBC1 is involved in adipocyte inflammation and is a possible marker of human adipose tissue senescence. Obesity 23 519-522. (https://doi.org/10.1002/ oby.20999)

Nin V, Chini CCS, Escande C, Capellini V \& Chini EN 2014 Deleted in breast cancer 1 (DBC1) protein regulates hepatic gluconeogenesis. Journal of Biological Chemistry 289 5518-5527. (https://doi. org/10.1074/jbc.M113.512913)

Rahman S \& Islam R 2011 Mammalian Sirt1: insights on its biological functions. Cell Communication and Signaling 9 11. (https://doi. org/10.1186/1478-811X-9-11) 
Richard AJ, Hang H \& Stephens JM 2017 Pyruvate dehydrogenase complex (PDC) subunits moonlight as interaction partners of phosphorylated STAT5 in adipocytes and adipose tissue. Journal of Biological Chemistry 292 19733-19742. (https://doi.org/10.1074/jbc. M117.811794)

Ruan H, Miles PDG, Ladd CM, Ross K, Golub TR, Olefsky JM \& Lodish HF 2002a Profiling gene transcription in vivo reveals adipose tissue as an immediate target of tumor necrosis factor-alpha: implications for insulin resistance. Diabetes 51 3176-3188 (https:// doi.org/10.2337/diabetes.51.11.3176)

Ruan H, Hacohen N, Golub TR, Van Parijs L \& Lodish HF 2002b Tumor necrosis factor-alpha suppresses adipocyte-specific genes and activates expression of preadipocyte genes in 3T3-L1 adipocytes: nuclear factor-kappaB activation by TNF-alpha is obligatory. Diabetes 51 1319-1336. (https://doi.org/10.2337/DIABETES.51.5.1319)

Stephens JM \& Pekala PH 1991 Transcriptional repression of the GLUT4 and C/EBP genes in 3T3-L1 adipocytes by tumor necrosis factoralpha. Journal of Biological Chemistry 266 21839-21845.

Stephens JM, Lee J \& Pilch PF 1997 Tumor necrosis factor-alpha-induced insulin resistance in 3T3-L1 adipocytes is accompanied by a loss of insulin receptor substrate-1 and GLUT4 expression without a loss of insulin receptor-mediated signal transduction. Journal of Biological Chemistry 272 971-976 (https://doi.org/10.1074/jbc.272.2.971)

Stewart WC, Pearcy LA, Floyd ZE \& Stephens JM 2011 STAT5A expression in Swiss 3T3 cells promotes adipogenesis in vivo in an athymic mice model system. Obesity 19 1731-1734. (https://doi. org/10.1038/oby.2011.66)

Story DJ \& Stephens JM 2006 Modulation and lack of cross-talk between signal transducer and activator of transcription 5 and suppressor of cytokine Signaling-3 in insulin and Growth Hormone signaling in 3T3-L1 adipocytes*. Obesity 14 1303-1311. (https://doi.org/10.1038/ oby.2006.148)
Teglund S, McKay C, Schuetz E, van Deursen JM, Stravopodis D, Wang D, Brown M, Bodner S, Grosveld G \& Ihle JN 1998 Stat5a and Stat $5 \mathrm{~b}$ proteins have essential and nonessential, or redundant, roles in cytokine responses. Cell 93 841-850. (https://doi.org/10.1016/ S0092-8674(00)81444-0)

Tse MCL, Liu X, Yang S, Ye K \& Chan CB 2013 Fyn regulates adipogenesis by promoting PIKE-A/STAT5a interaction. Molecular and Cellular Biology 33 1797-1808. (https://doi.org/10.1128/ MCB.01410-12)

Wakao H, Wakao R, Oda A \& Fujita H 2011 Constitutively active Stat5A and Stat5B promote adipogenesis. Environmental Health and Preventive Medicine 16 247-252. (https://doi.org/10.1007/s12199010-0193-7)

White UA, Coulter AA, Miles TK \& Stephens JM 2007 The STAT5Amediated induction of pyruvate dehydrogenase kinase 4 expression by prolactin or Growth Hormone in adipocytes. Diabetes $\mathbf{5 6}$ 1623-1629. (https://doi.org/10.2337/db06-1286)

White UA, Maier J, Zhao P, Richard AJ \& Stephens JM 2016 The modulation of adiponectin by STAT5-activating hormones. American Journal of Physiology: Endocrinology and Metabolism 310 E129-E136. (https://doi.org/10.1152/ajpendo.00068.2015)

Yu EJ, Kim S-H, Heo K, Ou C-Y, Stallcup MR \& Kim JH 2011 Reciprocal roles of DBC1 and SIRT1 in regulating estrogen receptor $\alpha$ activity and co-activator synergy. Nucleic Acids Research $396932-6943$. (https://doi.org/10.1093/nar/gkr347)

Zhao W, Kruse J-P, Tang Y, Jung SY, Qin J \& Gu W 2008 Negative regulation of the deacetylase SIRT1 by DBC1. Nature $\mathbf{4 5 1} 587-590$. (https://doi.org/10.1038/nature06515)

Zvonic S, Story DJ, Stephens JM \& Mynatt RL 2003 Growth Hormone, but not insulin, activates STAT5 proteins in adipocytes in vitro and in vivo. Biochemical and Biophysical Research Communications 302 359-362. (https://doi.org/10.1016/S0006-291X(03)00179-7)

Received in final form 30 July 2018

Accepted 23 August 2018

Accepted Preprint published online 23 August 2018 (c) 2018 Society for Endocrinology Published by Bioscientifica Ltd. Printed in Great Britain 